\title{
BOLIVIA: ¿ES POSIBLE LA CONSTRUCCIÓN DE UN NUEVo Estado? La Asamblea Constituyente y las Autonomías Departamentales
}

\author{
Bolivia: Is the Formation of a New State Possible? The Constituent \\ Assembly and the Departmental Autonomies.
}

\author{
GRACE IVANA DEHEZA* \\ Universidad Internacional de Florida
}

\begin{abstract}
RESUMEN
En 2007 se dieron en Boliva conflictos en torno a la Asamblea Constituyente, las autonomías y la asignación de los recursos del Estado. Las alineaciones de los diferentes actores políticos con respecto al tema de las autonomías demarcó la línea de división entre los aliados del gobierno y la oposición. Este trabajo, además de describir estos hechos, también muestra las tensiones entre los poderes del Estado, así como el desempeño económico y las relaciones exteriores establecidas por el gobierno del presidente Evo Morales.
\end{abstract}

Palabras clave: Asamblea Constituyente, autonomías, gobernabilidad, gobierno Evo Morales.

\begin{abstract}
In Bolivia during 2007 conflicts emerged in response to the Constituent Assembly, the autonomies, and the allocation of the State's resources. The alliances of the political actors in response to these issues defined the division between the government and opposition allies. In addition to describing these facts, this paper also analyzes the tensions among the executive, legislative and judiciary powers; and the economic performance and foreign relations established by the government of President Evo Morales.
\end{abstract}

Key words: Constituent Assembly, autonomy, governability, Evo Morales' Government.

\section{COYUNTURA POLÍTICA}

Los conflictos generados en torno a la Asamblea Constituyente (AC), las autonomías y la asignación de los recursos del Estado marcaron la coyuntura política de 2007. Los actores que impulsaron la AC y los que promovieron las autonomías en 2006 fueron los que tomaron las acciones e iniciativas para promover sus demandas ante la parálisis de los partidos tradicionales.

\footnotetext{
* La autora agradece los comentarios brindados por Eduardo Gamarra y Gabriela Hoberman en la elaboración
} de este trabajo. 
La ausencia de partidos organizados y estructurados a nivel nacional hizo que los grupos regionales se convirtieran en actores políticos con capacidad de movilización, inclusive mayor a la de los partidos con representación parlamentaria y con presencia en la AC. Estas fuerzas regionales asumieron la defensa de los intereses de sus departamentos, lo que les permitió crecer frente al colapso de las fuerzas políticas tradicionales. Los movimientos cívico-departamentales son, hasta el momento, los únicos que contrarrestan a nivel nacional la tendencia hacia el unipartidismo del gubernamental Movimiento al Socialismo (MAS).

Las alineaciones de los diferentes actores políticos con respecto a las autonomías demarcaron la línea de división entre los aliados del gobierno y la oposición. El gobierno identificó como sus adversarios a los prefectos ${ }^{1} \mathrm{y}$ a los líderes cívicos que enarbolaron las banderas autonomistas, y por ende, como a la oposición del MAS².

Los prefectos y comités cívicos de Santa Cruz, Beni, Pando y Tarija fueron los principales propulsores de las autonomías. Ellos pedían el respeto al referéndum del 2006 y la incorporación de su propuesta en la nueva Constitución. Los aspectos más relevantes de este proyecto eran: la consolidación de la elección directa del prefecto, el paso de las competencias en la toma decisiones de las políticas departamentales a las prefecturas, el cambio del sistema de asignación de los recursos en función a la generación de ingresos de cada departamento, la incorporación de un sistema de fondos de compensación solidarios con los departamentos más pobres, la administración de los recursos generados por algunos impuestos (el más polémico era el de hidrocarburos), y el traspaso de las competencias de agencias descentralizadas ${ }^{3}$. En definitiva, la propuesta rompía con el centralismo.

El gobierno era el impulsor de las autonomías indígenas ${ }^{4}$ paralelas a las departamentales y municipales. La continuidad cultural y lingüística indígena sería la base de la formación de estas autonomías. Los pueblos originarios tendrían la facultad de elegir y formar sus gobiernos autónomos según sus usos y costumbres, otorgándoles implícitamente un reconocimiento político de autodeterminación ${ }^{5}$. Los grupos originarios minoritarios podrían formar a su vez confederaciones de pueblos para dar paso a la formación de autonomías regionales. Los recursos naturales dentro de los territorios autónomos indígenas estarían bajo su administración. Estos pueblos tendrían la facultad de recaudar impuestos, además de recibir recursos del gobierno central.

1 Los prefectos fueron electos por primera vez junto con el presidente de la República en el año 2005, tienen una figura similar a la de los gobernadores, pero sin las atribuciones que les corresponden en los Estados federados.

2 Así lo manifestó el vicepresidente Álvaro García Linera en la primera reunión anual de evaluación de la gestión del gobierno con los movimientos sociales el 5 de enero del 2007. Extraído de Los Tiempos [En línea] <http://www.lostiempos.com/noticias/05-01-07/05_01_07_nac5.php> [Consulta: 04-01-2008]

3 Sobre este tema puede consultarse al autor de la propuesta autonomista de Santa Cruz: Juan Carlos Urenda, S.f. Separando la Paja del Trigo, Propuesta. Bases para construir las autonomías departamentales. Trabajo extraído de http://constituyentesoberana.org/info/files/SEPARANDO \%20LA\%20PAJA\%20DELTRIGO $\% 20$ Juan\%20Carlos\%20Urenda.pdf.

$4 \quad$ El vicepresidente era el impulsor del establecimiento de autonomías indígenas dentro de la nueva estructura político administrativa del país. Véase García Linera (2006a y 2006b).

5 Para un análisis crítico de esta propuesta véase Jorge Lazarte, 2007. ¿Multiculturalismo o multinacionalismo? Crítica a la propuesta del Movimiento al Socialismo. Manuscrito. 
Las diferencias entre ambas propuestas eran de fondo. Para los departamentos pro autonomía, la división político administrativa del país debería mantenerse. El principio ordenador sería el gobierno central, en un segundo nivel se encontrarían los gobiernos departamentales y en un tercer nivel estarían los gobiernos municipales. Las autonomías indígenas estarían dentro de los gobiernos departamentales. Para los cuatro departamentos, la propuesta del gobierno implicaba una reconfiguración del mapa político-administrativo del país, en el que se daría una superposición de competencias entre el gobierno departamental y el regional o indígena. Para el gobierno, la propuesta de los cuatro departamentos de oriente, más conocida como la Media Luna, implicaba en el fondo la división del país y el crecimiento desigual de los departamentos por la asignación presupuestaria. Estas dos visiones fueron las que imposibilitaron los acuerdos en la AC.

El primer conflicto de enero de 2007 en Cochabamba sería una muestra de la forma de reacción del gobierno y de sus seguidores ante las demandas autonomistas. El prefecto de Cochabamba, Manfred Reyes Villa, propuso en diciembre de 2006 convocar a un referéndum sobre la autonomía ${ }^{6}$ en ese departamento. En la primera quincena de enero se dieron los primeros enfrentamientos entre sectores ciudadanos afines al prefecto y los seguidores del MAS y el gobierno ${ }^{7}$. En esos enfrentamientos perdieron la vida un estudiante y un cocalero. Solamente, cuando el conflicto sufrió una escalada, el gobierno movilizó las Fuerzas Armadas y la Policía para retomar el control de esa ciudad ${ }^{8}$. Si bien el gobierno no pidió directamente la renuncia del prefecto, las bases gubernamentales en Cochabamba sí lo hicieron.

Las movilizaciones y presiones ejercidas desde la Media Luna y las presiones de los movimientos sociales afines al gobierno fueron permanentes durante todo 2007. Estas acciones han culminado en un empate catastrófico entre el gobierno y los prefectos. La solución de los temas de fondo ha planteado la necesidad de establecer un diálogo directo entre el gobierno central y los gobiernos departamentales para una creación de consensos sobre las autonomías que pueda englobar ambas propuestas ${ }^{9}$.

\section{Asamblea Nacional Constituyente}

En agosto de 2007 vencía el plazo para que la AC entregara el nuevo texto constitucional. Pero como se verá a continuación, las diferencias internas entre las fuerzas representadas en la AC y las presiones externas provenientes de los movimientos sociales, de las organizaciones cívicas, del gobierno central y de las prefecturas impidieron la elaboración de un texto que, al decir de Rousseau, respondiera a la voluntad general y no a la voluntad de todos.

En la AC existía una alta fragmentación debido a la presencia de 16 fuerzas entre partidos políticos, agrupaciones ciudadanas y pueblos indígenas. Cada fuerza tenía su propia

\footnotetext{
6 Tema y materia del referéndum del 2006. En ese departamento el No se impuso con $63,04 \%$ de los votos. Cocaleros, regantes, comerciantes y un sector de los universitarios.

Los Tiempos [En línea] <http://www.lostiempos.com/noticias/14-01-07/14_01_07_nac7.php.> . [Consulta: 04-01-2008].

9 Véase sección VIII.
} 
propuesta sobre el contenido de la nueva Constitución. Y en algunos casos las propuestas eran totalmente opuestas, lo que complicó la construcción de consensos. El partido mayoritario, el MAS, con 137 asambleístas, utilizó esta posición y su visión de país para articular una coalición mínima ganadora con las microrrepresentaciones para obtener los 170 votos necesarios para la aprobación en detalle de la nueva Constitución ${ }^{10}$. El segundo grupo mayoritario, Podemos, con 60 asambleístas, no logró establecer acuerdos con los representantes que podrían serle afines. El MNR, con todas sus facciones, reunía 18 asambleístas y la UN tenía ocho representantes, ambos partidos se constituían como pivotes necesarios en la conformación de un bloque que pudiera contrarrestar el peso del MAS en la asamblea. Sin embargo, Podemos no logró convocar a estos partidos. El MBL, que contaba con ocho representantes, era aliado del MAS. Las 23 restantes representaciones se encontraban dispersas en diez microorganizaciones; e incluso una parte trató de formar un grupo alternativo indígena como bloque de negociación ${ }^{11}$. Debido a las posiciones de cada fuerza y la fragmentación, la conformación de alianzas fue una tarea imposible, sobre todo en temas sensibles, como la visión de país, la autonomía y la propiedad de las tierras.

El MAS tuvo la dirección de once comisiones, Podemos, la de cuatro y el resto se distribuyó entre las representaciones menores. A semanas de vencer el plazo para la entrega del texto constitucional, las comisiones presentaron 194 artículos por unanimidad y sólo en tres de las 21 comisiones se presentó un solo informe por consenso ${ }^{12}$. Las restantes comisiones, por la falta de acuerdos entre la mayoría y la minoría, entregaron informes aprobados por ambas partes, inclusive la comisión de Visión de País presentó tres informes. En siete de las comisiones se incorporó la demanda de capitalidad plena para Sucre, que era el pedido del traslado de los poderes Ejecutivo y Legislativo a esa ciudad ${ }^{13}$. Así, al polémico tema de la autonomía se unió el tema de la capitalía, y ambos marcaron el desarrollo de los conflictos dentro de la AC y a nivel nacional.

La introducción de la capitalía en la agenda de la AC tenía repercusiones multiplicadoras. Inmediatamente surgieron presiones de La Paz y Chuquisaca. A ambas se alinearon de distinta forma los siete departamentos y el Poder Ejecutivo, originándose dos grupos: los que apoyaban la demanda de Chuquisaca y los que respaldaban a La Paz.

10 La mayoría era necesaria para aprobar el texto constitucional en grande, y el MAS la tenía. Pero para la aprobación en detalle se necesitaban 170 miembros contando la totalidad de los asambleístas. En términos estrictos, el MAS necesitaba contar con este número o con la indisciplina de los asambleístas para sesionar con el quórum reglamentario de 128 miembros y así aprobar la nueva Constitución. La fragmentada oposición sólo podía formar coaliciones bloqueadoras para evitar la aprobación del texto constitucional.

11 Se utiliza este nombre genérico para designar a los partidos políticos, pueblos indígenas y organizaciones de la sociedad civil.

12 Las comisiones de Desarrollo Humano, Estructura del Nuevo Estado y Tierra. En las últimas dos habían observaciones que debían ser analizadas en el plenario.

13 A fines del siglo XIX, La Paz era el centro de la economía del neo Estado Boliviano, y Sucre donde había nacido la República, era la capital. El ímpetu del Partido Liberal por trasladar el gobierno a La Paz resultó en el surgimiento un movimiento federalista en La Paz, Oruro y Santa Cruz. La revolución federal, como se conoce a esta etapa de la historia de Bolivia, trasladó el centro político y el gobierno de Sucre a La Paz, pero no se estableció una forma de Estado Federal. Para mayores referencias sobre el tema véase Isaac Sandoval Rodríguez (1987). 
El tema salió de la AC a la presión en las calles en forma de cabildos. En estas reuniones multitudinarias se demostraba la capacidad de movilización social de cada departamento. En la ciudad de El Alto de La Paz se realizó un cabildo bajo la consigna "la sede no se mueve". Por su parte, las organizaciones cívicas de Chuquisaca organizaron otro cabildo en la ciudad de Sucre. Los prefectos de Santa Cruz, Beni, Pando y Tarija apoyaron a Chuquisaca, con lo que la Media Luna obtuvo así un aliado circunstancial contra el centralismo. Este conflicto avivó el clivaje regional en el país. El Presidente sugirió que los temas que atentaban contra la unidad nacional no deberían tocarse en la $\mathrm{AC}^{14}$.

Las dos posiciones tenían consecuencias políticas. Por un lado, el bloque pro capitalía fortalecía a la oposición al gobierno y, por otro lado, el bloque que se oponía al traslado de poderes conflictuaba la relación entre los aliados del gobierno y el MAS. Dentro del partido del presidente, el tema de la capitalía atravesaba las líneas partidarias. Esta agrupación tenía doce representantes de Chuquisaca, que apoyaban la demanda de su región, pero también tenía 32 representantes de La Paz, que se oponían al traslado de los poderes. El tema dividía también a los aliados de Evo Morales, el MBL apoyaba a la capitalía y el Movimiento sin Miedo (MSM) ${ }^{15}$ apoyaba la sede de La Paz. El MAS estaba bajo doble presión: La Paz presionaba por no tocar este tema y los representantes de Chuquisaca del MAS y del MBL apoyaban las propuestas de capitalía. Ante esta situación, quedaban sólo dos posibilidades: sacar el tema de la capitalía de la agenda de la AC o cerrarla, lo que sería un fracaso para el MAS y para el gobierno. En una sesión en la que se utilizó el quórum reglamentario, los asambleístas del MAS decidieron excluir el tema de la capitalía del debate. En medio de tensiones, sectores afines al gobierno se movilizaron a Sucre para presionar por la conclusión del trabajo de la AC. En vista de los posibles enfrentamientos entre los sectores movilizados, el prefecto de Chuquisaca renunció a su cargo ${ }^{16}$. Paralelamente y en la ciudad de La Paz, los miembros del MAS redactaban un proyecto de constitución donde se incluían temas controversiales tales como la reelección indefinida para presidente y vicepresidente, la existencia de una sola cámara y la gestión territorial autónoma indígena. El 6 de septiembre se producen los primeros enfrentamientos entre sectores afines al MAS y los sectores cívicos de Sucre.

Estos enfrenamientos paralizaron a la AC y ante el virtual colapso de la misma, el vicepresidente de las República convocó a un diálogo para la solución del conflicto y conformó un consejo político de negociación con el Comité Interinstitucional de Chuquisaca, el Comité de Emergencia de La Paz y el gobierno, bajo el liderazgo de Álvaro García Linera. Tras una serie de negociaciones, el consejo propuso a las entidades cívicas de Chuquisaca el traslado de la Corte Nacional Electoral, y el Registo Civil, así como el establecimiento de nuevas instituciones estatales en Sucre, propuesta que rechazó el Comité chuquisaqueño. La reacción del vicepresidente fue inmediata. En un mensaje al país enfatizó que "La Constituyente está secuestrada, chantajeada y presionada por una alianza circunstancial de esas tres minorías (aludiendo a Podemos, a los empresarios y a 
algunos dirigentes chuquisaqueños)... [La] Asamblea está en manos de la Constituyente, pero fundamentalmente queda en manos del pueblo boliviano, que confiamos sabrá encontrar el mecanismo para liberar a la Constituyente de este chantaje ultra conservador de reaccionarios racistas y fascistas de algunos sectores empresariales y políticos que quieren hacer daño al país"17.

La ciudad de Sucre no era más un lugar de encuentros, sino de enfrentamientos. La polarización de fuerzas llegaba al extremo. Los ponchos rojos, un sector aliado al MAS, en el pueblo altiplánico de Ayo Ayo del departamento de La Paz decapitaron a dos perros como una forma de intimidación a los opositores del gobierno. Al mismo tiempo, sectores de El Alto afines al MAS y al gobierno se trasladaron a Sucre para presionar por la aprobación del nuevo texto constitucional. El 24 de noviembre, los asambleístas del MAS sesionaron en un cuartel militar cercano a la ciudad de Sucre bajo el resguardo de sectores movilizados afines al MAS, el partido del presidente aprobó en grande el texto ${ }^{18}$ de la Constitución con 136 votos, sin la participación de asambleístas de la oposición ${ }^{19}$. Mientras tanto, residentes de Sucre salieron a protestar para evitar que la AC sesionara, produciéndose enfrentamientos en el que perdieron la vida dos civiles.

Los asambleístas finalmente deberían aprobar en detalle el texto. Para el MAS y el gobierno, el imperativo era trasladar la AC a otro lugar que le permitiera sesionar. La única forma legal de hacerlo era la sanción de una ley por el Congreso. El 28 de noviembre, sectores afines al MAS realizaron literalmente un cerco al Congreso Nacional en la Plaza Murillo, impidiendo el acceso a parlamentarios de la oposición. En la cámara baja, y con la participación de senadores suplentes, se aprobó el traslado de las sesiones de la AC. Una vez trasladadas las sesiones a Oruro y en una sesión maratónica en la que asistieron únicamente 162 miembros de la Asamblea, se aprobó en detalle lo que ya estaba escrito, es decir la Nueva Constitución Política del Estado (NCPE). Un referéndum nacional deberá aprobar ahora la nueva Carta Magna ${ }^{20}$.

La AC, que tuvo el reto de construir una Constitución que respondiera a la diversidad existente, había fracasado. La normativa, el grupo mayoritario y la presión se impusieron al diálogo y a la concertación.

Los prefectos de la oposición, a los que se unió el de La Paz, manifestaron su discrepancia y desconocieron el nuevo texto constitucional. Mientras tanto, Evo Morales convocó a celebrar la NCPE y los prefectos de los cuatro departamentos de Santa Cruz, Beni, Pando y Tarija se declararon autónomos. El sábado 15 de diciembre, cada sector realizó sus propios festejos bajo un clima de tensión política. Las demostraciones ponían en evidencia el empate entre

17 Extraído Los Tiempos [En línea] <http://www.lostiempos.com/noticias/07-11-07/07_11_07_nac8.php> [Consulta: 7-11-2007 y archivada].

18 Existieron discrepancias entre el texto propuesto y el aprobado. [En línea] <http://www.laprensa.com.bo/ noticias/29-11-07/29_11_07_poli1.php> [Consulta: 29-11-2007 y archivada].

19 Véase Los Tiempos 25 de noviembre de 2007. Para lograr que se diera el quórum reglamentario, las fuerzas de la oposición lo lograron ingresar al cuartel militar.

20 Es importante mencionar que a través de la Radio se informó que el asambleísta Jorge Lazarte quiso abandonar la sesión pero no pudo. También doce asambleístas de Chuquisaca quisieron abandonar la sesión. Sin embargo, fueron convencidos por integrantes del MAS para permanecer y aprobar el nuevo texto constitucional. 
ambos grupos. El presidente una vez más pidió medirse con los prefectos y llamar a un referéndum revocatorio, para así salir de la situación en la que se encontraba el país.

La polarización entre el gobierno y los prefectos llegó a su punto más alto en momentos que la ciudadanía se disponía a celebrar la Navidad. La distensión de Bolivia se logró por las fiestas de fin de año y por la convocatoria al diálogo entre las fuerzas en conflicto. Las expectativas frente al diálogo son altas, ya que podría lograr lo que la AC no logró: conciliar posiciones opuestas que sean favorables para todos los bolivianos ${ }^{21}$.

\section{COYUNTURA SOCIAL}

Durante 2007, el gobierno presionó por las demandas regionales y sectoriales. En cada caso, el Ejecutivo tuvo que entablar negociaciones y acceder a las peticiones para evitar el escalamiento de las manifestaciones $y$, especialmente, que se diera una convergencia de los conflictos. ${ }^{22}$

En los municipios intermedios surgieron conflictos por el control de las alcaldías, como fue el caso de Yapacaní, en el departamento de Santa Cruz. Por el control de la alcaldía de este municipio se enfrentaron sectores del MAS contra la Confederación de Colonizadores de la región.

A causa de la falta de la delimitación de competencias entre el gobierno central y departamental, se originaron conflictos en las regiones. En algunos casos, los manifestantes tomaron acciones de hecho. En Yacuiba y en Villamontes de Tarija, los locatarios tomaron Pocitos y San Antonio para manifestarse contra la falta de atención a su demanda por la solución de los límites territoriales entre las provincias del Chaco y $\mathrm{O}^{\prime} \mathrm{Connor}$. El gobierno y la prefectura se acusaban mutuamente por la falta de solución del conflicto.

A nivel regional provincial, el gobierno también enfrentó oposición a la implementación de sus políticas. En Yacuiba los vecinos, campesinos y ganaderos se opusieron a la política de dotación de tierras del gobierno central. Todos estos hechos demuestran la necesidad de una normativa que regule las competencias del gobierno central y de los gobiernos departamentales en lo referente a la política de reasignación de tierras, sin la cual los conflictos que surgieron en Yacuiba son sólo un ejemplo de los que podrían darse posteriormente en Bolivia.

Para finalizar esta sección, es importante destacar que el discurso de Evo Morales y del MAS ha redefinido lo que es el pueblo y la nación. Para él, la centralidad de la nación está en los pueblos originarios, a través de la cual se integran todos los demás sectores de la sociedad que no se definen bajo el concepto indígena $u$ originario, como son las

21 Cuando se terminaba de escribir el presente trabajo, Gobierno y prefectos de la oposición se sentaron a una mesa de diálogo abierto, que se transmite por el canal de televisión y radio estatal.

22 Según Laserna, ya en los primeros 14 meses de gobierno de Evo Morales el número promedio de conflictos superó a la mayoría de los gobiernos democráticos desde 1982 -a excepción de Siles Zuazo y de Mesa-, con 38,5 eventos mensuales $(2007,109)$. 
clases medias; los otros son las fuerzas conservadoras que se oponen a los $\operatorname{cambios}^{23}$. La fuerza interpelativa de los discursos masistas, de Evo Morales y de Álvaro García Linera ha movilizado a los movimientos sociales por la defensa de los intereses del pueblo y de la democracia contra las fuerzas que ellos consideran conservadoras.

\section{COYUNTURA ECONÓMICA}

Los indicadores económicos en el segundo año de la gestión del presidente Evo Morales muestran comportamientos mixtos. Según las proyecciones de la CEPAL el crecimiento del Producto Interno Bruto para el 2007 será de un 3,8\%, levemente menor al crecimiento que se registró el año anterior. ${ }^{24}$ La contracción del PIB se dio durante el primer trimestre debido a la menor producción del sector agropecuario y de la minería. El fenómeno natural de "El Niño" produjo pérdidas para el sector agropecuario calculadas en 138 millones de dólares. La producción del sector minero cayó por los conflictos entre los sectores cooperativistas y asalariados de Huanuni.

Entre los aspectos positivos, se encuentra el superávit de la balanza de pagos. Según datos oficiales del Banco Central de Bolivia (BCB), de enero a septiembre de 2007 el saldo fue de 1.472 millones de dólares, cifra ligeramente menor a la registrada en el 2006. Esto se debe a varios factores. El primero, los incrementos de las transferencias provenientes del extranjero, o las remesas de trabajadores bolivianos que se encuentran en el extranjero. De enero a septiembre, las remesas aportaron 650,9 millones de dólares, siendo un 69,5\% más para el mismo periodo del año pasado ${ }^{25}$. El segundo, el incremento de los ingresos por los intereses recibidos de las reservas internaciones; durante el periodo analizado ascienden a 208,7 millones de dólares. La exportación de minerales, y de soya y sus derivados contribuyeron positivamente a la balanza de pagos, gracias al incremento de los precios, ya que los volúmenes de las exportaciones de ambos bajaron.

Otro de los indicadores positivos fue la disminución de la tasa de desempleo abierta, que fue de $9,5 \%,{ }^{26}$ a pesar de la falta de inversión privada nacional y extranjera en los sectores productivos capaces de absorber la mano de obra. El crecimiento del empleo se dio en los sectores de la construcción, de servicios financieros y de la minería.

Entre los aspectos negativos está la inflación. Por primera vez después de 22 años, el índice de precios al consumidor creció por encima de los dos dígitos. Ya a principios de 2007 se registraron presiones inflacionarias debido al incremento de los productos de la canasta básica familiar, lo que fue percibido por el gobierno como especulación ${ }^{27}$. Para la oposición, bo/prensa/Noticias.asp?id=200707264\&p=5.

24 Siendo la segunda más baja de América del Sur. CEPAL, Balance preliminar de las economías de América Latina y el Caribe 2007, diciembre 2007. Pág. 103.

25 Reporte de Balanza de Pagos.

26 2007: Crecimiento de la Economía y Generación del Empleo en Bolivia. CEDLA, mayo, Año 4, No 6.

27 El Ministro de Hacienda inclusive sugirió no comprar aquellos productos que registraron incrementos de precios. 
la inflación era causada por el incremento del dinero ocasionado por la falta de control del circuito coca cocaína del narcotráfico, como manifestó Samuel Doria Medina ${ }^{28}$.

Algunos expertos en materia económica consideraban que los síntomas de la inflación eran producto del incremento de los salarios y del gasto público ${ }^{29}$ y por lo tanto, de una falta de austeridad. Otros analistas consideraban que las presiones inflacionarias eran una manifestación del síndrome Holandés debido al incremento de ingresos del país por el volumen de las remesas provenientes del exterior ${ }^{30} \mathrm{y}$ del ingreso de circulante por la cooperación venezolana ${ }^{31}$.

El gobierno y las autoridades del BCB tomaron recién en el segundo semestre medidas para controlar la masa monetaria y bajar los precios de algunos productos básicos, para así frenar la inflación ${ }^{32}$. Sin embargo, estas medidas no lograron revertir las tendencias, y la inflación acumulada a noviembre llegó al 11,02\%,33 la tercera inflación más alta de América Latina y el Caribe después de Venezuela y Nicaragua (CEPAL: 2007, 175).

Otro factor negativo es la caída de la inversión directa neta externa. De enero a septiembre fue de 57 millones de dólares, mientras que para el mismo período del año pasado la misma llegó a 95,9 millones de dólares.

El sector de hidrocarburos es el más afectado, ya que desde el 2003 las inversiones fueron mínimas. Como consecuencia, la cadena productiva se estancó y no pudo satisfacer la demanda interna ni cumplir con los contratos de exportación a Brasil y a Argentina. Por el desabastecimiento del gas, las presiones de los consumidores nacionales e internacionales llegaron al gobierno, que priorizó el mercado interno para evitar la especulación, el contrabando y frenar así a tiempo las protestas en las calles.

El proceso de nacionalización y la incertidumbre que generaba la Asamblea Constituyente desalentó la inversión nacional, ${ }^{34}$ especialmente por la garantía de los derechos sobre la propiedad privada y de la tierra para la producción agropecuaria. También la Confederación de Empresarios Privados de Bolivia (CEPB) continuamente se lamentó por la falta de atención a las necesidades de este sector y de incentivos para promover la inversión en el país.

La Razón [En Línea] <http://www.la-razon.com/Versiones/20070710_005963/nota_248_451365.htm >. [Consulta 10-7-2007 y archivada].

29 Como lo expresara tempranamente el ex presidente del Banco Central de Bolivia en un artículo de opinión publicado en: La Razón [En línea] <http://www.la-razon.com/versiones/20070504_005896/nota_248_422691. htm > . [Consulta 5-4-2007 y archivada].

30 Para el economista Ramiro Martín Luján, La Razón[En línea]<http://www.la-razon.com/versiones/20070905_006020/ nota_246_475781.htm>. [Consulta 5-9-2007 y archivada].

31 Aspecto que será analizado más adelante.

32 Las medidas adoptadas fueron la autorización a las AFP para invertir en el exterior, la venta de títulos del Estado, la apreciación del boliviano frente al dólar, el incremento del porcentaje del encaje marginal en moneda extranjera, la importación directa de productos (harina) y la creación de una Empresa de Apoyo a la Producción de Alimentos para los sectores micro productores.

33 INE, Resumen Estadístico Mensual. Número 11, Año 2007. Pág. 1.

34 Declaraciones dadas por el presidente de la CEPB a la Prensa el día 8 de octubre de 2007. 
Tabla 1: Indicadores Económicos Bolivia.

\begin{tabular}{lcccc}
\hline & \multicolumn{4}{c}{ Tasa de Variación en \% } \\
\cline { 2 - 5 } & 2004 & 2005 & 2006 & 2007 \\
\hline Indicadores Económicos* & 3,9 & 4,0 & 4,6 & 3,8 \\
PIB & - & 1,8 & 2,5 & 1,7 \\
PIB por Habitante & 4,6 & 4,9 & 4,9 & 11,9 \\
Precios al Consumidor & $-4,2$ & $-5,1$ & 4,5 & -1 \\
Variación del Salario Mínimo & & & & \\
Real & & & & \\
& & & 3.863 & 3.139 \\
Algunos indicadores de la Balanza de Pagos** & 3.280 & 2.809 & 2.428 \\
En millones del \$US & 2.362 & 2.865 & 278 & 55 \\
$\begin{array}{l}\text { Exportaciones de bienes } \\
\text { Importaciones de bienes FOB }\end{array}$ & 2.146 & 182 & & \\
Inversión extranjera directa & 1.725 & & & \\
neta FOB & & & & \\
\hline
\end{tabular}

Fuentes: *Banco Central de Bolivia $2007 \mathrm{y}{ }^{* *}$ Cepal 2007 y 2006.

\section{COYUNTURA INTERNACIONAL}

Evo Morales continuó la política de la Diplomacia de los Pueblos bajo los principios de igualdad y reciprocidad con los que inició su gestión. Con aquellos presidentes regionales autoidentificados de izquierda, considerados amigos y aliados, el gobierno afianzó sus vínculos de cooperación. El mejor ejemplo es el de Venezuela. En contraste a este tipo de negociaciones, el gobierno pragmáticamente mantuvo la cooperación económica con otros países, como es el caso de los Estados Unidos.

Con Venezuela, el gobierno logró varios acuerdos importantes entre los que se destaca la conformación de Petroandina, consorcio entre PEDEVESA y YPFB dedicado a la exploración y explotación de recursos de hidrocarburos en el territorio nacional, aunque hasta la fecha no se ha dado ninguna inversión en este sector.

El gobierno recibió de Venezuela donaciones económicas directas para la implementación del programa "Bolivia Cambia, Evo Cumple", donaciones reguladas por el DS 29097 de marzo del 2007. Según el presidente Morales, las donaciones que entraron a este programa fueron de 55,3 millones de dólares ${ }^{35}$. Los conocidos "cheques" venezolanos llegaron a los municipios que solicitaran fondos de acuerdo a convocatoria pública ${ }^{36}$. Bajo una modalidad

35 Mensaje a la Nación del presidente Evo Morales Ayma, Sucre 6 de agosto del 2007. Versión Online <http:// abi.bo/index.php?i=enlace\&j=documentos/discursos/200708/06-08-2007-discurso.htm>

36 El uso de esos fondos no estuvo exento de problemas, en el caso de un municipio rural en el altiplano de La Paz donde se denunció la malversación de fondos que deberían ser investigados bajo la Ley SAFCOLey ( ${ }^{\circ}$ 1178). 
similar, la asistencia llegó a las Fuerzas Armadas y a la Policía Nacional. Los fondos deberían utilizarse para mejorar en la infraestructura y para la dotación de equipos.

La estrecha relación entre Hugo Chávez y Evo Morales ha generado un respaldo del presidente venezolano al presidente boliviano, en el que el primero sale a la defensa del líder aymara aun cuando se considere como una injerencia en los asuntos internos del país $^{37}$.

Estos lazos de amistad entre ambos países permitieron en varias oportunidades que aviones militares venezolanos se desplazaran y permanezcan en el territorio boliviano, como revelaron canales de televisión y la prensa independiente. Estos fueron hechos que molestaron a la oposición. En especial, la presencia del presidente venezolano Hugo Chávez en territorio nacional con el consentimiento del gobierno y sin la aprobación del Congreso, molestó al ex presidente Jorge Quiroga ${ }^{38}$.

El gobierno del presidente Morales avanza paulatinamente en la creación de confianza con Chile. Desde la puesta en marcha de la agenda sin exclusiones con trece puntos, ambos países han llevado a cabo acciones de cooperación e integración que generan un nuevo clima de cordialidad entre ambos gobiernos. Por primera vez desde la llamada "guerra del Gas", el gobierno boliviano, a través de su canciller David Choquehuanca, abrió la posibilidad de la venta de gas, si eso conllevara a una salida al $\operatorname{mar}^{39}$. El legendario tema que congeló las relaciones entre ambos países ahora es parte de la agenda. Para el Palacio Quemado y para La Moneda, el acceso al océano Pacífico y la restauración de relaciones diplomáticas son temas sensibles que no se debaten públicamente, sino que se realizan en las más altas esferas de ambos gobiernos ${ }^{40}$. Cualquier indiscreción ha sido objeto de reacciones inmediatas de ambas partes, como ocurrió con la destitución del cónsul de Bolivia en Chile, Roberto Finot, tras haber realizado declaraciones a la prensa sobre la posible restauración de relaciones diplomáticas entre ambos países.

Este proceso de integración se potenció aún más en diciembre, con el acuerdo de la construcción de un corredor bioceánico entre Brasil, Bolivia y Chile. Acuerdo que también sirvió para dar un giro a las relaciones con Brasil, que estuvieron deterioradas por la nacionalización de los hidrocarburos, los precios del gas y la compra de las refinerías de Petrobrás.

Las relaciones con Planalto se han refrescado con la firma del convenio tripartito. Evo Morales e Inacio Lula Da Silva sellaron acuerdos para reimpulsar la inversión de Petrobrás en la exploración de campos gasiferos para el abastecimiento del gas a Brasil. Con este acuerdo, Evo Morales ganó un aliado internacional con alta credibilidad. El presidente

37 Hugo Chávez en su conocido programa Aló Presidente amenazó a "la oligarquía boliviana” asegurando que "el gobierno venezolano no se quedaría con las manos cruzadas" y que defendería al presidente Morales "si lo derrocaran o asesinaran... se instalaría un Vietnam de las ametralladoras". Declaraciones que fueron consideradas por el gobierno como una muestra de solidaridad, mientras para la oposición eran una muestra de la injerencia directa en los asuntos internos del país.

38 Véase <http://www.lostiempos.com/noticias/13-11-07/13_11_07_nac15.php> [Consulta 13-11-2007 y archivada].

39 En declaraciones dadas a la prensa durante la conmemoración del aniversario de la Pérdida del Mar.

40 Según la Cancillería Boliviana. 
Lula Da Silva en su estadía en la ciudad de La Paz le dio su apoyo y respaldo al gobierno de Morales, pero como él mismo manifestara, "sin inmiscuirse en la política interna de Bolivia".

El gobierno de Evo Morales decidió establecer relaciones políticas y comerciales con Irán, al argumentar la capacidad de desarrollo tecnológico y de la transferencia de ese Know How a Bolivia; a pesar de los antecedentes en los temas derechos humanos y de construcción material nuclear que tiene dicho país a nivel internacional.

Con Estados Unidos, las relaciones han sido tensas. La polémica sobre la producción de los cultivos de coca enfrían y tensionan las relaciones entre la Embajada de Estados Unidos y el Palacio Quemado ${ }^{41}$. Pero por otro lado, la cooperación hacia algunos sectores, inclusive en materia de lucha contra el narcotráfico, se considera como positiva ${ }^{42}$.

A pesar de la retórica anticapitalista y antiimperialista del presidente Morales ${ }^{43}$ su gobierno adoptó posiciones pragmáticas a la hora de recibir la cooperación de Estados Unidos y de buscar la renovación de la APTDEA ${ }^{44}$. Diferentes misiones gubernamentales se dirigieron a Washington para sensibilizar sobre la importancia de la ampliación de este acuerdo comercial para Bolivia ${ }^{45}$. Sin embargo, debido al clima de las relaciones entre ambos países resulta complicada la renovación de este acuerdo en el Congreso norteamericano.

La necesidad de inversiones en los sectores primarios, minería e hidrocarburos, ha generado una diplomacia pragmática para captar la inversión internacional. En el sector minero se obtuvieron capitales provenientes de la India, Estados Unidos y Canadá.

En definitiva, el gobierno adoptó posiciones ideológicas y pragmáticas. Con los países miembros del ALBA continuó con su política de cooperación. Pero también adoptó una posición pragmática al continuar con la cooperación económica de otros países, como es el caso de Estados Unidos, y de inversión de ese mismo país, Canadá y la India.

\section{PODER EJECUTIVO}

Cumplido el primer año de gobierno, el presidente Evo Morales realizó en enero la primera recomposición de su gabinete ministerial, al nombrar a siete nuevos ministros y ratificar a nueve. Más tarde, en agosto, durante las efemérides patrias, ratificó a todo su gabinete

41 En agosto del 2007 el representante de Estados Unidos en Bolivia, Philip Golberg, afirmó que en la actual gestión "hubo un incremento en el cultivo de coca y que también había un "poco más de narcotráfico". Estas afirmaciones causaron molestias en el gobierno nacional, el embajador Golberg fue llamado por la cancillería Boliviana a dar explicaciones sobre sus afirmaciones http://www.lostiempos.com/noticias/23-08-07/23_08_07_nac3. php

42 Los Tiempos [En línea] <http:/ / www.lostiempos.com/noticias/27-08-07/nacional.php> . [Consulta 27-8-2007 y archivada].

43 Discurso del presidente Evo Morales ante las Naciones Unidas 24 de septiembre de 2007. Extraído de <http: / / abi.bo/index.php?i=discursos $>$. [Consulta 28-12-2007].

44 Por sus siglas en inglés el "Andean Trade Promotion and Drug Eradication Act" es el acuerdo de preferencia arancelaria para los productos provenientes de la región Andina hacia Estados Unidos.

45 A tiempo de escribir este trabajo y faltando dos meses para el vencimiento de este acuerdo entre ambos países aún no existe ninguna resolución del Congreso de Estados Unidos. 
de ministros por la alineación y ejecución de las directrices del gobierno contenidas en el Plan Nacional de Desarrollo, a pesar de que integrantes de los movimientos sociales pidieron el cambio de los de Educación, Trabajo y Aguas.

Durante este año se realizaron tres cambios de ministros: los de Educación, Obras Públicas y Aguas. En el primer caso, se sacó a Víctor Cáceres de su cargo por haber dado declaraciones que iban en contra de los lineamientos generales de las políticas antineoliberales del gobierno; a lo que se le sumó la incapacidad de solucionar un conflicto con el sector del magisterio. En el segundo, Jerjes Mercado Suárez renunció a su cargo y en el tercero, se sacó a Abel Mamani de su cargo por los escándalos públicos en los que estuvo envuelto. Las nuevas nominaciones recayeron sobre personas con capacidades técnicas y sin vínculos estrechos con los movimientos sociales o sindicales.

La composición actual del gabinete ministerial es técnico independiente (véase Tabla 2), a diferencia del primer gabinete de Morales, que contaba con representantes de sectores sindicales. Es notable que en estos dos años los ministros que respondían a sectores sindicales o sociales se hayan cambiado casi en la totalidad y no se sustituyeron por otras personalidades de esos sectores. Estos cambios, sin duda, le han dado una mayor capacidad de decisión interna, fuera de las presiones de los sectores sociales representados en el gabinete ministerial ${ }^{46}$.

A pesar que el presidente cambió en el lapso de dos años a la mitad de su equipo ministerial, existe una estabilidad en los ministerios de: Presidencia, Hidrocarburos, Defensa y Relaciones Exteriores. A este grupo de ministerios se le puede considerar parte del grupo estratégico para la administración del Estado ${ }^{47}$. Estos ministros son los más cercanos al presidente y, como se verá más adelante, él les ha ratificado su confianza cuando la cámara de senadores les dio un voto de censura. Los ministerios de los sectores productivos y sociales permanecen mientras exista una continuidad y confluencia de las acciones desarrolladas en esos ministerios y el Plan de Desarrollo de Evo Morales.

El funcionamiento interno del Ejecutivo responde a todas las características de un presidencialismo fuerte y jerárquico ${ }^{48}$. Las reuniones del gabinete ministerial están dirigidas por el presidente, y en ellas también participa el vicepresidente García Linera, pese a que formalmente no es parte del gabinete. Estas reuniones le permiten al presidente tener un control gubernamental sobre la correspondencia de las políticas con los lineamientos del Plan Nacional. Evo Morales ha implementado un sistema de evaluación semestral y anual en el que se reúne el gabinete ampliado: ministros y viceministros. En el último, en diciembre, también participaron dirigentes de los movimientos sociales con el propósito de obtener apoyo para la aprobación del nuevo texto constitucional.

Dos casos ejemplifican esto. El Ministerio de Educación no estuvo asignado inicialmente a ningún "representante" de los sectores de la educación, llámese sindicatos de maestros urbanos o rurales. Cuando Evo Morales reemplazó a Víctor Cáceres por Magdalena Cajías tuvo quejas de esos sectores sindicales pero no hubo reclamos por la nominación de un representante de ese sector. Por el contrario, en la destitución del Ministro de Aguas Abel Mamani, la FEJUVE y otros sectores sindicales de El Alto reclamaron para sí la designación de un dirigente o representante de esa ciudad.

47 Sobre la definición de las áreas de gobiernos, véase Deheza 1997, 122.

48 Sobre este tema, véase Blondel 2007. 
Tabla 2: Equipo Ministerial: Toma de Juramento 23 de enero del 2007.

\begin{tabular}{|c|c|c|}
\hline Ministerio & Nombre & Cambio Ministerial \\
\hline Ministro de la Presidencia & $\begin{array}{l}\text { Juan Ramón Quintana. } \\
\text { Independiente ( } 23 \text { de enero de } \\
\text { 2006) }\end{array}$ & \\
\hline Ministro de Defensa & $\begin{array}{l}\text { Walker San Miguel Rodríguez. } \\
\text { Independiente ( } 23 \text { de enero de } \\
\text { 2006) }\end{array}$ & \\
\hline Ministro de Gobierno & $\begin{array}{l}\text { Alfredo Rada Vélez ( } 23 \text { de enero } \\
\text { de 2006) }\end{array}$ & \\
\hline Ministro de Hacienda & $\begin{array}{l}\text { Luis Alberto Arce Catacora. } \\
\text { Independiente ( } 23 \text { de enero de } \\
\text { 2006) }\end{array}$ & \\
\hline $\begin{array}{l}\text { Ministro de Relaciones Exteriores y } \\
\text { Culto }\end{array}$ & $\begin{array}{l}\text { David Choquehuanca. } \\
\text { Independiente ( } 23 \text { de enero de } \\
\text { 2006) }\end{array}$ & \\
\hline $\begin{array}{l}\text { Ministro de Desarrollo Sostenible y } \\
\text { Planificación, posteriormente llamado } \\
\text { Ministerio de Planificación del } \\
\text { Desarrollo }\end{array}$ & $\begin{array}{l}\text { Gabriel Loza Tellería. Independiente } \\
\text { ( } 23 \text { de enero de 2007) }\end{array}$ & \\
\hline $\begin{array}{l}\text { Ministra de Desarrollo Económico y } \\
\text { Microempresa }\end{array}$ & $\begin{array}{l}\text { Nilda Sosa Luna (Dirigente } \\
\text { sindical) ( } 23 \text { de enero de 2006) }\end{array}$ & \\
\hline Ministro de Servicios y Obras Públicas & $\begin{array}{l}\text { Jerjes Mercado Suárez ( } 23 \text { de enero } \\
\text { de 2007) }\end{array}$ & $\begin{array}{l}\text { José Kimm Franco. } \\
\text { Independiente (7 de } \\
\text { septiembre de 2007) }\end{array}$ \\
\hline Ministro de Hidrocarburos & Carlos Villegas, el 19 de septiembre & \\
\hline Ministro de Educación & $\begin{array}{l}\text { Víctor Cáceres Rodríguez. Dirigente } \\
\text { sindical ( } 23 \text { de enero de 2007) }\end{array}$ & $\begin{array}{l}\text { Magdalena Cajías. } \\
\text { Independiente ( } 9 \text { de } \\
\text { junio de 2007) }\end{array}$ \\
\hline Ministra de Salud & $\begin{array}{l}\text { Nila Heredia. Independiente ( } 23 \text { de } \\
\text { enero de 2006) }\end{array}$ & \\
\hline Ministro de Trabajo & $\begin{array}{l}\text { Walter Juvenal Delgadillo (ex- } \\
\text { militante del MIR-Masas)( } 23 \text { de } \\
\text { enero de 2007) }\end{array}$ & \\
\hline $\begin{array}{l}\text { Ministro de Desarrollo Rural, } \\
\text { Agropecuario y Medio Ambiente }\end{array}$ & $\begin{array}{l}\text { Susana Rivero Guzmán (23 de } \\
\text { enero de 2007) }\end{array}$ & \\
\hline Ministra de Justicia & $\begin{array}{l}\text { Celina Torrico Rojas ( } 23 \text { de enero } \\
\text { de 2007) }\end{array}$ & \\
\hline Ministro del Agua & $\begin{array}{l}\text { Abel Mamani, dirigente sindical ( } 23 \\
\text { de enero de 2006). Destituido }\end{array}$ & $\begin{array}{l}\text { Walter Valda. } \\
\text { Asume funciones } \\
\text { el } 28 \text { de noviembre } \\
\text { 2007) }\end{array}$ \\
\hline Ministro de Minería y Metalurgia & $\begin{array}{l}\text { Asume Guillermo Dalence, el } 6 \text { de } \\
\text { octubre del } 2006\end{array}$ & $\begin{array}{l}\text { Es sustituido por } \\
\text { Luis Alberto Echazú } \\
\text { el } 28 \text { de marzo } 2007\end{array}$ \\
\hline
\end{tabular}

Fuente: (La Razón y la Prensa) Recomposición ministerial del 23 de enero del 2007 y cambios ministeriales realizados durante el 2007. 
El presidente manifestó en muchas ocasiones que había lentitud en el Congreso para aprobar los proyectos de ley del Ejecutivo. Por esto, una gran parte de las políticas las ha implementado a través de decretos supremos. Desde enero hasta diciembre de 2007, el Poder Ejecutivo emitió 382 decretos, cifra menor a la registrada en la gestión de 2006, cuando se emitieron 1.078.

El gobierno siguió con las políticas de bienestar social, promovió el Bono Solidario de 2.400 bolivianos al año a todos los mayores de 60 años que no cuenten con jubilación. Desde los ministerios continuó con los programas de alfabetización y salud.

\section{PODER LEGISLATIVO}

En la Cámara de Diputados, el MAS es mayoritario (véase Tabla 3). Las relaciones entre esta cámara y el Ejecutivo son prioritarias. La primera ha adaptado su agenda según las necesidades del segundo. Es por esto que la cámara baja aprobó con celeridad todas las propuestas de leyes originadas en el Poder Ejecutivo y de la misma forma las remitió al Senado para su consideración.

En la cámara alta, el MAS es minoritario, al perder la dirección del Senado en la legislatura del 2007. El bloque integrado por Podemos, el MNR y la UN viabilizó la elección de José Villavicencio, representante de UN, como presidente de esta cámara ${ }^{49}$. Esta correlación de fuerzas permitió a la oposición ejercer su capacidad de veto a los proyectos de ley aprobados en la Cámara de Diputados, ya sea retrasándolos o aprobándolos, pero con modificaciones. Estas acciones han desatado conflictos entre el Senado y el Ejecutivo. El último considera que éste es un obstáculo para la implementación de sus políticas. Según Evo Morales, 97 proyectos estarían a la espera de la aprobación del Senado.

El MAS, a pesar de su situación minoritaria en el Senado, ha utilizado más de una vez el recurso del quórum reglamentario para pasar las propuestas de leyes aprobadas en la Cámara de Diputados. Utilizando este reglamento, el MAS aprovechó la ausencia de los miembros de la oposición y con senadores suplentes aprobó la propuesta de ley para que la AC pudiera sesionar en cualquier parte del territorio nacional ${ }^{50}$.

49 Extraído de Los Tiempos [En línea] < http:/ / www.lostiempos.com/noticias/25-01-07/nacional.php> [Consulta 01-25-2007 y archivada].

$50 \quad$ Ley 3792 del 28 de noviembre de 2007. 
Tabla 3: Composición del Congreso Nacional.

\begin{tabular}{lcc}
\hline \multicolumn{1}{c}{ Partidos } & Diputados & Senadores \\
\hline Movimiento al Socialismo (MAS) & 72 & 12 \\
Poder Democrático y Social (PODEMOS) & 43 & 13 \\
Frente de Unidad Nacional (UN) & 8 & 1 \\
Movimiento Nacionalista Revolucionario (MNR) & 7 & 1 \\
Total & 130 & 27 \\
\hline
\end{tabular}

Fuente: Corte Nacional Electoral.

La Cámara de Senadores trató de establecer mecanismos de control al Poder Ejecutivo, cada senador solicitó en promedio 11.1 informes escritos, mientras que en la Cámara de Diputados, cada representante solicitó en promedio 7.8 informes escritos ${ }^{51}$. En el Senado, se llegaron a censurar a tres ministros de Estado: David Choquehuanca, Juan Ramón Quintana y Alfredo Rada. Los dos primeros, por no dar respuesta a las peticiones de informes sobre el caso de Walter Chávez; y en último caso, por las muertes acaecidas en la ciudad de Sucre durante los conflictos de noviembre. Ninguno de los tres ministros fue destituido. Más bien, el presidente los ratificó en sus cargos.

Tabla 4: Composición Directivas del Congreso gestión 2007.

\begin{tabular}{lll}
\hline \multicolumn{1}{c}{ Cargo } & \multicolumn{1}{c}{ Cámara de Senadores } & \multicolumn{1}{c}{ Cámara de Diputados } \\
\hline Presidencia & José Villavicencio (UN). & Edmundo Novillo (MAS) \\
Primer Vicepresidente & Carlos Borht (Podemos) & Nemesia Chacollo (MAS) \\
Segundo Vicepresidente & Guido Guardia (MAS) & Katia Romero (Podemos) \\
Primer Secretario & Fernando Rodríguez (Podemos) & Filemón Aruni (MAS) \\
Segundo Secretario & Tito Carrazana (MAS) & Orlando Miranda (MAS) \\
Tercer Secretario & Paulo Bravo (Podemos) & Jorge Becerra (MAS) \\
Cuarto Secretario & Esta cámara no tiene cuarto & Roxana Sandoval (MNR) \\
\hline
\end{tabular}

Fuente: Para la Cámara de Senadores Los Tiempos 25 de enero de 2007 y para la Cámara de Diputados La Razón 21 de enero del 2007.

\section{PODER JUDICIAL}

Durante 2007 se dieron conflictos entre los poderes Ejecutivo y Judicial, que inclusive involucraron al Senado, por la designación de cuatro ministros de la Corte Suprema de Justicia. Esta nominación es prerrogativa del Poder Legislativo, pero el presidente

51 Legislativos $\mathrm{N}^{\circ}$ 4, La Paz. Pág. 2. [En Línea] <http:/ / www.populi.org.bo/r107/04-fiscalizacion.pdf> . [Consulta 28 de diciembre 2007]. 
la realizó para cubrir las vacancias en esa institución. Amparados en esa prerrogativa, los senadores de la oposición presentaron ante el Tribunal Constitucional un recurso de inconstitucionalidad al alegar que las designaciones rompían con el principio de independencia y balance de poderes y, al mismo tiempo, mostraban la intención del gobierno de cooptar al Poder Judicial. El fallo a favor de los demandantes desató la indignación del gobierno y promovió un juicio de responsabilidades en el Congreso a los cuatro miembros del Tribunal Constitucional. En una sesión, la Cámara de Senadores liberó de todas las acusaciones a los magistrados del Tribunal Constitucional. Sin embargo, las magistradas Elizabeth Iñiguez de Salinas y Martha Rojas Álvarez renunciaron a sus cargos por las constantes acusaciones de algunos miembros de la Cámara de Diputados ${ }^{52}$. Dos meses más tarde, en diciembre, lo haría el presidente Walter Raña dejando al Tribunal Constitucional sin quórum para su funcionamiento.

Para el nuevo año se espera que el Congreso realice las nuevas designaciones del Poder Judicial.

\section{POST DATA}

En el momento de cerrar este trabajo, en la segunda semana de enero del 2008 se dieron dos hechos importantes. El primero, el presidente Evo Morales, el vicepresidente y el círculo técnico de ministros y viceministros más próximo a él iniciaron un diálogo con los prefectos de los departamentos opositores para llegar a un acuerdo nacional sobre: las autonomías, la asignación de los recursos del Estado y la NCPE; a estos se les añade los referéndums revocatorios para presidente y prefectos. Los resultados de este diálogo aún están por verse.

El segundo, el nombramiento del nuevo vocal de la Corte Nacional Electoral (CNE). Las relaciones entre Evo Morales y la CNE fueron tensas desde su inicio. Cuando Morales resultó electo presidente en las urnas, criticó el accionar de la CNE por la depuración del padrón electoral. En su discurso de victoria en Cochabamba pidió la dimisión de los vocales de esta institución. El presidente de la CNE, Óscar Hassenteufel, renunció a su cargo a los tres meses del ascenso de Evo Morales. No obstante, la CNE llevó adelante las elecciones para la AC y para el referéndum autonómico bajo la presidencia de Salvador Romero Ballivián.

El nuevo vocal José Luis Exeni es un intelectual reconocido por su trabajo como periodista y politólogo. El gran temor de parte de la oposición es que esta elección tenga una inclinación hacia el MAS. La oposición ve con recelo la posición de la CNE en los referendos revocatorios que se avecinan. La voluntad política de mantener imparcial de esta institución se medirá cuando en el Congreso designe a los otros dos vocales que faltan.

52 Jornada [En línea] < http://www.jornadanet.com/Bolivia/n.php?a=200710271043181> . [Consulta 28 de diciembre 2007]. 
La complicada situación y el nivel de conflictos en el país ha producido el declive en la popularidad del presidente Evo Morales, según una encuesta de opinión de Mora y Araujo publicada en Los Tiempos el 11 de diciembre. Para ese mes, la popularidad del presidente se encontraba en un $46 \%$, frente a marzo anterior, en que obtuvo un $60 \%$. La encuesta también revela que un 23,9\% de los entrevistados considera que la NCPE responde a las necesidades del MAS. Y que el 77\% de los entrevistados le echa la culpa al gobierno por los conflictos del país. Con estos resultados de la opinión pública, el futuro y la permanencia del presidente, vicepresidente y los prefectos se decidirán en el referéndum revocatorio. Este 2008 se abre con mucho optimismo por el diálogo entre el gobierno y los prefectos de la oposición, pero también existe una incertidumbre de lo que podría suceder si el diálogo fracasara.

\section{REFERENCIAS}

Asamblea Constituyente de Bolivia. Constitución Política del Estado. Aprobada en Grande. Sucre, 24 de noviembre de 2007. Versión en línea obtenida del ABI.

Asamblea Constituyente de Bolivia. Constitución Política del Estado. Aprobada en Grande, detalle y revisión. [En línea] <http://abi.bo/coyuntura/asamblea/nueva_cpe_aprobada_en_grande_en_detalle_y_ en_revision.pdf > [Consulta: 28-12-2007].

Banco Central de Bolivia. 2007. Reporte de la Balanza de Pagos y Posición de la Inversión Internacional. Enero - Septiembre.

Blondel, Jean. 2007. Comparing cabinet life in presidential and parliamentary systems: towards an empirical analysis. Mimeo.

Cletus Gregor Barié. 2007. Bolivia: ¿Cuánta revolución cabe en la democracia? Fundación para las Relaciones Internacionales y el Diálogo Exterior. Marzo. Versión En línea.

CEDLA, 2007. Alerta laboral. Año 6, octubre.

Cámara Nacional de Industrias. 2007. Evolución de la Economía y de la Industria Nacional. Presentación de Power Point. Septiembre.

CEPAL. 2007. "Bolivia". Balance preliminar de las economías de América Latina y el Caribe 2007. Santiago: CEPAL.

CEPAL. 2006. "Bolivia". Estudio económico de América Latina y el Caribe 2006-2007. Santiago: CEPAL.

De Jong, Niek y Juan Carlos Aguilar. 2007. Informe País 2006 Bolivia: Gobernar con los Movimientos Sociales. Estocolmo: ASDI.

Deheza, Grace I. 2007. “Bolivia 2006: Reforma Estatal y Construcción del Poder". Revista de Ciencia Política 27 (EE): 43-57.

Deheza, Grace I. 1997. Coaliciones de Gobierno en el Sistema Presidencial: América del Sur. Tesis doctoral no publicada, Instituto Universitario Europeo, Florencia Italia.

Estatuto del Departamento Autónomo de Santa Cruz. Aprobado en sus tres etapas, en grande, detalle y revisión. Santa Cruz, 15 de diciembre de 2007. [En línea] <http://www.eldeber.com.bo/2007/2007-12-15/ autonomia.php> [Consulta: 28-12-2007].

Gamarra, Eduardo A. 2007. Bolivia on the Brink. Washington: Council on Foreign Relations.

García, L. Álvaro. 2006a. "Lo Nacional-Popular en Acción: El Evismo". El Juguete Rabioso. (Abril.) Págs. 8-9.

García Linera, Alvaro. 2006b "Autonomías regionales indígenas y Estado multicultural". In: La descentralización que se viene. La Paz: FES-ILDIS.

Instituto Nacional de Estadística. 2007. Resumen Estadístico Mensual. $\mathrm{N}^{\circ} 11,07$.

Hemisphere. 2006. Volumen 17, Otoño. En prensa. Edición dedicada a Bolivia. 
Hoberman, Gabriela. 2007. "Ethnic Politics in a Divided Country: Bolivia and the Logic of Identities". Tesis de Maestría presentada al Departamento de Ciencia Política de la Universidad Internacional de la Florida.

Laserna, Roberto. 2007. "El Caudillismo Fragmentado". Nueva Sociedad No 209, mayo-junio de 2007, ISSN: 0251-3552, [En línea] <www.nuso.org>.

Natason, José. 2007. "Las Reformas Pactadas: Entrevista con Álvaro García Linera”. Nueva Sociedad No 209 (mayo-junio). Págs. 161-172.

PNUD. 2007. Informe Nacional sobre Desarrollo Humano 2007: El estado del Estado en Bolivia. La Paz: PNUD.

Roncagliolo, Rafael y Carlos Meléndez. 2007. La Política por Dentro Cambios y Continuidades en las Organizaciones Políticas de los Países Andinos. Estocolmo: Idea.

Stahler-Sholk, Richard, Harry E. Vanden, y Glen David Kuecker. 2007. "Introduction Globalization Resistance: The New Politics of Social Movements in Latin America". Latin American Perspectives 5 (Marzo). Pág. 5-15.

Tapia Mealla, Luis. 2006. La invención del núcleo común. Ciudadanía y gobierno multisocietal. La Paz: CIDES - UMSA. [En línea] <http://bibliotecavirtual.clacso.org.ar/ar/libros/bolivia/cides/tapia.pdf.> [Consulta: 28-12-2007].

Urenda Díaz, Juan Carlos. 2005. Separando la Paja del Trigo. [En línea] <http://constituyentesoberana. org/info/files/SEPARANDO\%20LA\%20PAJA\%20DELTRIGO\%20Juan\%20Carlos\%20Urenda.pdf>. [Consulta: 28-12-2007].

Weyland, Kart. 2007. Politics and Policies of Latin America's Two Lefts: The Role of Party Systems vs. Resource Bonanzas. Paper presentado al XXVI Congreso de LASA, Montreal 5 al 9 de septiembre 2007.

Grace Ivana Deheza Obtuvo su título de Ph.D. en Ciencia Política en el Instituto Universitario Europeo, Florencia, Italia. Actualmente es docente adjunta del programa de Maestría de Ciencia Política en Español de la Universidad Internacional de Florida en Miami, y es consultora de Newlink Research.

(E-mail: gideheza@bellsouth.net). 
